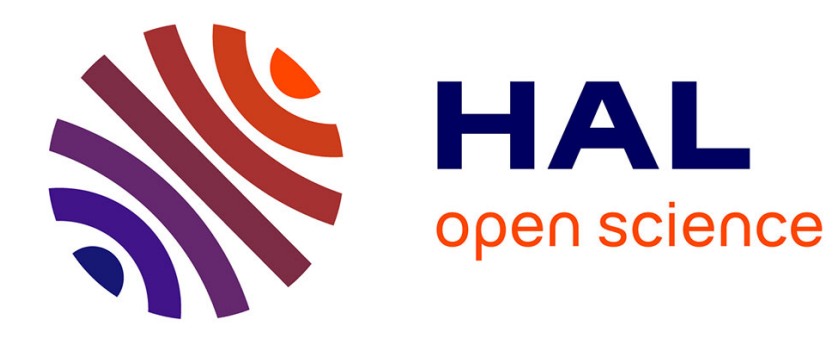

\title{
A new random color filter array with good spectral properties
}

\author{
Laurent Condat
}

\section{To cite this version:}

Laurent Condat. A new random color filter array with good spectral properties. Actes de IEEE ICIP, Nov 2009, Le Caire, Egypt. pp.1613 - 1616, 10.1109/ICIP.2009.5413678 . hal-00814076

\section{HAL Id: hal-00814076 https://hal.science/hal-00814076}

Submitted on 16 Apr 2013

HAL is a multi-disciplinary open access archive for the deposit and dissemination of scientific research documents, whether they are published or not. The documents may come from teaching and research institutions in France or abroad, or from public or private research centers.
L'archive ouverte pluridisciplinaire HAL, est destinée au dépôt et à la diffusion de documents scientifiques de niveau recherche, publiés ou non, émanant des établissements d'enseignement et de recherche français ou étrangers, des laboratoires publics ou privés. 


\title{
A NEW RANDOM COLOR FILTER ARRAY WITH GOOD SPECTRAL PROPERTIES
}

\author{
Laurent Condat*
}

\author{
GREYC \\ 6 Bd du Maréchal Juin \\ 14050 CAEN Cedex, France
}

\begin{abstract}
We propose in this work a new random pattern with $\mathrm{R}, \mathrm{G}, \mathrm{B}$ colors, which can be used to design color filter arrays (CFA) with good spectral properties, since the chrominance is modulated at high frequencies. Hence, the demosaicking artifacts appear as incoherent noise, which is less visually disturbing than the moiré structures characteristic of periodic CFAs.
\end{abstract}

Index Terms - Color filter array, random pattern, blue noise, demosaicking

\section{INTRODUCTION}

Digital cameras acquire color images by means of a sensor on which a color filter array (CFA) is overlaid. The most popular is the Bayer CFA, which consists in red (R), green (G) and blue (B) filters arranged periodically [1]. Given the mosaicked image acquired by the sensor, the demosaicking operation is required to reconstruct a full color image with complete R,G,B components, see e.g. [1, 2, 3]. Actually, very few works have addressed the design of new CFAs with improved performances over the Bayer CFA [4, 5, 6, 16], known to yield moiré artifacts in certain conditions. In fact, whatever the CFA, aliasing in unavoidable, because of the intrinsic undersampling conditions of the acquisition. However, it is known, especially for printing [7, 8] and computer graphics applications [9], that random sampling yields images where aliasing appears as incoherent noise instead of more disturbing moiré artifacts $[10,11]$. Motivated by this property, we propose in this work a new design of CFAs with R,G,B filters arranged in a non-periodic pattern.

Previous work has shown how to characterize the spectral representation induced by the mosaicking process [2]. Based on them, we justify in Section 2 the choice of CFAs whose color channels have blue noise characteristics; that is, a low concentration of energy around the zero frequency. R,G,B sampling with a random blue noise pattern is also a good model for the human visual system [12].

\footnotetext{
* Part of this work was performed during the stay of the author in the Helmholtz Zentrum München, supported by the Marie Curie Excellence Team Grant MEXT-CT-2004-013477, Acronym MAMEBIA, funded by the European Commission. Contact: laurent. condategreyc. ensicaen.fr.
}

We describe our approach in Section 3 and evaluate its performances in Section 4 using three generic demosaicking algorithms. As a result, the demosaicking artifacts are more visually pleasant than with the Bayer CFA or other periodic CFAs.

\section{SPECTRAL PROPERTIES OF CFAS}

\subsection{Spectral Characterization of Mosaicking}

In this article, boldface letters denote vectors, e.g. $\mathbf{k}=$ $\left[k_{1}, k_{2}\right]^{\mathrm{T}} \in \mathbb{Z}^{2}$ and $\mathbf{0}=[0,0]^{\mathrm{T}}$. A CFA with R,G,B filters is a color image $\mathbf{c f a}=(\mathbf{c f a}[\mathbf{k}])_{\mathbf{k} \in \mathbb{Z}^{2}}$, where $\mathbf{c f a}[\mathbf{k}] \in$ $\{\mathbf{R}, \mathbf{G}, \mathbf{B}\}$ is the color of the filter at location $\mathbf{k} \in \mathbb{Z}^{2}$, and $\mathbf{R}=[1,0,0]^{\mathrm{T}}, \mathbf{G}=[0,1,0]^{\mathrm{T}}, \mathbf{B}=[0,0,1]^{\mathrm{T}}$. The mosaicked image $v=(v[\mathbf{k}])_{\mathbf{k} \in \mathbb{Z}^{2}}$ acquired by the sensor is such that $v[\mathbf{k}]=\mathbf{i m}[\mathbf{k}]^{\mathrm{T}} \mathbf{c f a}[\mathbf{k}]$ for every $\mathbf{k} \in \mathbb{Z}^{2}$, where the color image $\mathbf{i m}=(\mathbf{i m}[\mathbf{k}])_{\mathbf{k} \in \mathbb{Z}^{2}}$ is the ground truth to be estimated by demosaicking. We define the Fourier transform of a signal $g=(g[\mathbf{k}])_{\mathbf{k} \in \mathbb{Z}^{2}}$ as $\hat{g}(\boldsymbol{\omega})=\sum_{\mathbf{k} \in \mathbb{Z}^{2}} g[\mathbf{k}] e^{-j \boldsymbol{\omega}^{\mathrm{T}} \mathbf{k}}$.

It is well known that, in natural images, the R,G,B components are not independent $[13,1]$. Thus, we consider instead the orthonormal basis corresponding to luminance, red-green (R-G) and blue-yellow (B-Y) chrominance, defined as

$\mathbf{L}=\frac{1}{\sqrt{3}}[1,1,1]^{\mathrm{T}}, \mathbf{C}_{1}=\frac{1}{\sqrt{2}}[-1,1,0]^{\mathrm{T}}, \mathbf{C}_{2}=\frac{1}{\sqrt{6}}[-1,-1,2]$

We denote $g^{L}, g^{C_{1}}$, and $g^{C_{2}}$ the components of a color signal $\mathrm{g}$ in this basis. These components can be considered statistically independent for natural images [13].

Alleysson et al. showed that the mosaicked image $v$ can be interpreted, in the Fourier domain, as the sum of the luminance and chrominance components of the color reference image im, moved at different locations of the frequency plane [2]. In our notations, from $v[\mathbf{k}]=\operatorname{im}^{L}[\mathbf{k}] \operatorname{cfa}^{L}[\mathbf{k}]+$ $\operatorname{im}^{C_{1}}[\mathbf{k}] \operatorname{cfa}^{C_{1}}[\mathbf{k}]+\operatorname{im}^{C_{2}}[\mathbf{k}] \operatorname{cfa}^{C_{2}}[\mathbf{k}]$ and $\operatorname{cfa}^{L}[\mathbf{k}]=1 / \sqrt{3}$ for every $\mathbf{k}$, we get ( $*$ denotes the convolution):

$$
\hat{v}(\boldsymbol{\omega})=\frac{1}{\sqrt{3}} \widehat{\operatorname{im}^{L}}(\boldsymbol{\omega})+\sum_{C \in\left\{C_{1}, C_{2}\right\}} \widehat{\operatorname{im}^{C}}(\boldsymbol{\omega}) * \widehat{\operatorname{cfa}^{C}}(\boldsymbol{\omega})
$$




\subsection{The Goal of CFA design}

Since the signals $\mathrm{im}^{L}, \mathrm{im}^{C_{1}}$ and $\mathrm{im}^{C_{2}}$ are lowpass, the best way for reducing the overlap between the chrominance and luminance signals, which is the cause of most of the artifacts in the demosaicked image [2], is to reject the chrominance in the corners of the Nyquist band, around the frequency $\boldsymbol{\pi}=(\pi, \pi)$. Thus, our target is a CFA with the energy of $\mathrm{cfa}^{C_{1}}$ and $\mathrm{cfa}^{C_{2}}$ concentrated around $\pi$. Note that $\widehat{\mathrm{cfa}^{C_{1}}}$ and $\mathrm{cfa}^{C_{2}}$ can not both be Dirac distributions at $\pi$. For a periodic CFA, $\widehat{\mathrm{cfa}^{C_{1}}}$ and $\widehat{\mathrm{cfa}^{C_{2}}}$ are sums of Dirac distributions, located on the dual lattice of the spatial lattice underlying the periodicity of the CFA [6]. This limitation does not hold for aperiodic patterns. This observation is our motivation for seeking a CFA with a random pattern and chrominance having blue noise characteristics; that is, minimal energy in the baseband.

In the case of the Bayer CFA, the chrominance is located in the frequency plane at the frequencies $\pi,[0, \pi]^{\mathrm{T}},[\pi, 0]^{\mathrm{T}}$ and 0 [2]. The presence of chrominance energy at $[0, \pi]^{\mathrm{T}}$ and $[\pi, 0]^{\mathrm{T}}$ is responsible for the moiré artifacts that appear in areas of the image with horizontal or vertical high-frequency content. Moreover, a CFA without preferred color axis (that is, with one third of R, of G, and of B filters) is better, since the luminance is not corrupted by chrominance in the baseband of $v$ and can be optimally recovered during demosaicking.

\subsection{Blue Noise Patterns}

Poisson disk distributions are blue noise patterns that can be defined as the limit of a uniform sampling process with a minimum-distance rejection criterion $[14,11]$. In our context, we define the minimum-distance criterion as the property that two adjacent pixels have different colors. This ensures that low-frequency content in the chrominance is avoided in an optimal way. To our knowledge, only the work of Zhu and Parker address the problem of designing random R,G,B patterns with blue noise characteristics, using blue noise masks thresholded at different levels [5]. This method does not yield patterns with good spectral characteristics, however: the small example [5, Fig. 18 b)] clearly shows clusters of adjacent pixels with the same color.

\section{FAST RANDOM TILING}

Our method, which is very fast, consists in filling in the mosaic in scanline order, in two steps:

1. The first row of the mosaic is generated in scanline order, by adding successively tiles of three pixels chosen among the following six tiles:

$$
\begin{array}{lll}
1:[R, G, B] & 2:[R, B, G] & 3:[G, B, R] \\
4:[G, R, B] & 5:[B, R, G] & 6:[B, G, R]
\end{array}
$$

Each new tile is chosen randomly among two possible tiles, depending on the tile at its left. The admissible adjacent combinations are:

$$
\begin{array}{lll}
1 \rightarrow\{2,4\}, & 2 \rightarrow\{1,5\}, & 3 \rightarrow\{4,6\} \\
4 \rightarrow\{1,3\}, & 5 \rightarrow\{2,6\}, & 6 \rightarrow\{3,5\} .
\end{array}
$$

For example, at the right of a tile 1, we have the choice between the tiles 2 and 4 .

The first column of the mosaic is generated the same way, using vertical 3-tiles.

2. The mosaic is then filled in in scanline order. Each pixel receives a color different from the ones of its left and top neighbors. If two colors are possible, we assign the color different from the one of the top-left diagonal neighbor. So, this second step is deterministic.

Using this method, we obtain an aperiodic tiling of the whole image with tri-colored diamonds, as illustrated in Fig. 1 (a). The choice of using 3-tiles is empirical, but has been found to yield the best results among numerous other methods tried. For reproducibility purpose, Matlab code generating the random pattern has been made available online ${ }^{1}$.

The amplitude spectrum of the B-Y chrominance component for the pattern in Fig. 1 (a) is given in Fig. 1 (b). It was obtained by smoothing the amplitude of the FFT with a $5 \times 5$ box filter. The R-G chrominance channel has the same spectrum, up to random fluctuations. We can observe how the energy is concentrated in the area $[-\pi, \pi]^{2} \backslash[-2 \pi / 3,2 \pi / 3]^{2}$. Hence, our design yields a pattern with blue noise characteristics, as expected. We believe that there still exists some margin of improvement over the proposed design, with a better concentration of the energy of the chrominance around $\pi$. Actually, there must be some intrinsic balance between this goal and the amount of energy around $\mathbf{0}$. Indeed, energy at $\boldsymbol{\pi}$ corresponds to local bi-colored checkerboards in the pattern, which also have energy at $\mathbf{0}$ (colored DC component).

\section{PERFORMANCE ANALYSIS}

In order to validate our designs experimentally, we considered the same classical data set of 20 color images, like in [6]. These images were mosaicked using the proposed and the Bayer CFAs and demosaicked using three different methods:

1. We propose a simple linear scheme that consists in computing a missing value for the color $\mathbf{C} \in$ $\{\mathbf{R}, \mathbf{G}, \mathbf{B}\}$ at location $\mathbf{k}$, by averaging the pixel values $v[\mathbf{l}]$ for $\mathbf{l}$ in a $3 \times 3$ neighborhood surrounding $\mathbf{k}$ and for which $h[\mathbf{l}]=\mathbf{C}$. This simple scheme reverts to bilinear interpolation for the Bayer pattern.

\footnotetext{
${ }^{1}$ See the homepage of the author: http://www.greyc. ensicaen.fr/ Icondat/publications.html
} 


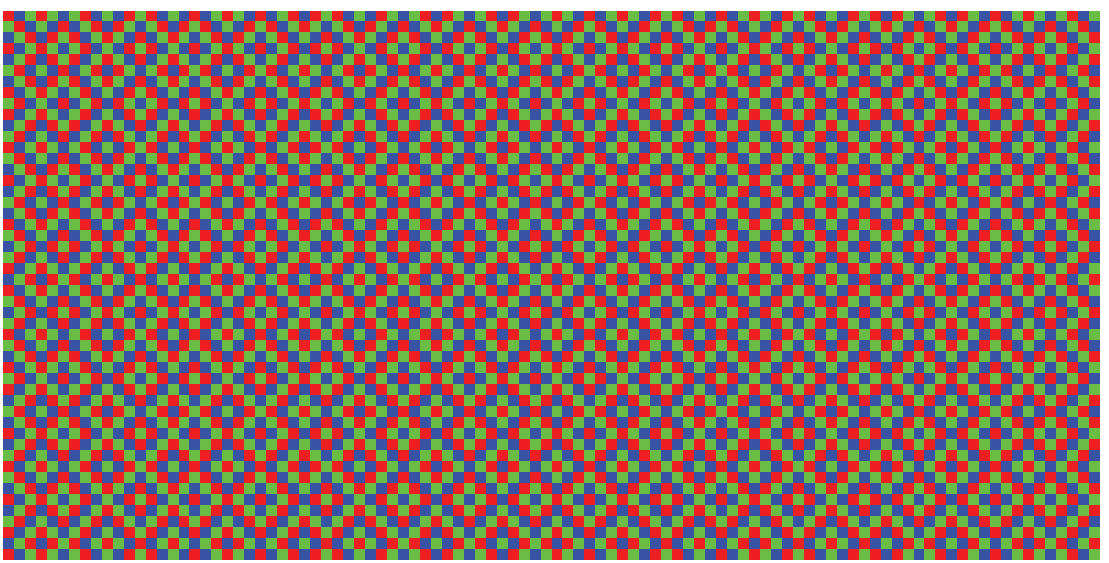

(a)

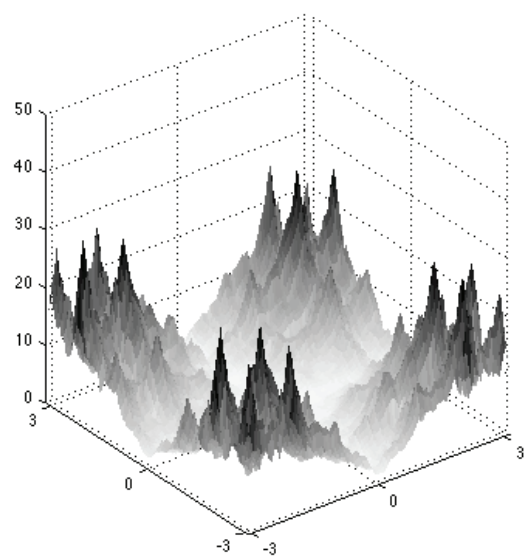

(b)

Fig. 1. (a) Part of the mosaic of size $100 \times 100$ generated by the algorithm described in Section 3 and (b) the (smoothed) magnitude of the FFT for the B-Y chrominance of the mosaic.

2. We implemented the non-linear universal demosaicking algorithm of Lukac et al. [3] which is, to our knowledge, the only demosaicking algorithm proposed in the literature, that can be used for every R,G,B CFA.

3. We developed a new linear demosaicking algorithm, that can be applied to every CFA [15]. It is based on a variational framework: the image with minimal smoothess interpolating the data is computed.

The numerical results for every combination of CFAs and demosaicking methods are summarized in Tab. $1^{2}$. We first observe that the simple linear demosaicking method, that does not exploit the cross-correlations between the color bands in natural images, provides relatively poor results. Our random CFA outperforms the Bayer CFA by a small margin. The demosaicking method of Lukac yields a much better quality. The best performances are obtained with the Bayer CFA, but there might be a bias if this method has precisely been tuned to perform well with this CFA in particular. The new method developed in [15] performs globally best, although the one of Lukac remains better for the Bayer CFA only. Our new CFA yields better performances with this third method, too. Note that the first and third demosaicking methods are linear; hence, their results are more reliable indications of the intrinsic quality of the CFAs under test.

A visual inspection of the demosaicked images allows to observe the appearance of the aliasing artifacts characteristic of each CFA. Fig. 2 shows the fence in the lighthouse image, often used as benchmark for comparing demosaicking results. In fact, due to the superposition of the chrominance and luminance in the mosaicked image according to Eqn. (2), the demosaicking algorithm has to assign a given information either

\footnotetext{
${ }^{2}$ We do not take into account the first and last three rows and columns of the demosaicked images for the computation of the MSE, since the initial images used for the tests have been badly acquired at the boundaries.
}

\begin{tabular}{c|ccc||ccc|} 
& \multicolumn{3}{|c||}{ Bayer } & \multicolumn{3}{c|}{ Proposed CFA } \\
& bilinear & {$[3]$} & {$[15]$} & bilinear & {$[3]$} & {$[15]$} \\
\hline MSE & 93.39 & 12.19 & 12.87 & 90.07 & 17.96 & $\mathbf{1 1 . 9 6}$
\end{tabular}

Table 1. Average mean square error over the 20 test images for the demosaicking experiments of Section 4 using the different combinations of CFAs and demosaicking methods.

to the chrominance or to the luminance of the demosaicked image. In case of a bad decision, artifacts appear [2]. If energy corresponding to high frequency luminance information is assigned to the chrominance, color fringes or chrominance noise appear. The artifacts present with the random CFA are, to our point of view, much more visually pleasant than the structured fringes characteristic of the Bayer CFA. Although the demosaicking method of [3] provides lower MSE for the Bayer pattern, the artifacts are less visible with our CFA.

\section{CONCLUSION}

We proposed a new method for generating R,G,B CFAs with random patterns, such that two adjacent pixels have different colors. This property ensures that the chrominance channels have reduced energy in the low frequency area, a necessary property to minimize aliasing. In comparison with the Bayer pattern, the demosaicking artifacts are reduced both quantitatively and qualitatively. This superiority also holds over the other periodic R,G,B arrangements compared in [4], as reported in [15]. In practice, a small random pattern (e.g. of size $18 \times 18$ ) can be designed with our method and periodically replicated to tile the whole CFA. Such a pseudo-random pattern is more easily realizable and yields almost the same performances as a true random pattern.

It was shown recently that strong improvements can be obtained with CFAs having colors other than R,G,B $[6,16]$. 
This makes the physical realizability of the CFA more involved, however. Actually, the key contribution of the present work is the non-periodic random pattern with appealing spectral properties. CFA design is one application, but this work may be of interest for, e.g., multitone dithering in printing [17] or texture generation in computer graphics [18].

\section{REFERENCES}

[1] B. K. Gunturk, J. Glotzbach, Y. Altunbasak, R. W. Schaffer, and R. M. Mersereau, "Demosaicking: Color filter array interpolation," IEEE Signal Processing Mag., vol. 22, no. 1, pp. 44-54, Jan. 2005.

[2] D. Alleyson, S. Süsstrunk, and J. Herault, "Linear demosaicing inspired by the human visual system," IEEE Trans. Image Processing, vol. 14, no. 4, pp. 439-449, Apr. 2005.

[3] R. Lukac and K. N. Plataniotis, "Universal demosaicking for imaging pipelines with a RGB color filter array," Pattern Recognition, vol. 38, pp. 2208-2212, 2005.

[4] _ - "Color filter arrays: Design and performance analysis," IEEE Trans. Consumer Electron., vol. 51, no. 4, pp. 12601267, Nov. 2005.

[5] W. Zhu, K. Parker, and M. A. Kriss, "Color filter arrays based on mutually exclusive blue noise patterns," Journal of Visual Communication and Image Representation, vol. 10, no. 3, pp. 245-267, Sept. 1999.

[6] K. Hirakawa and P. J. Wolfe, "Spatio-spectral color filter array design for optimal image recovery," IEEE Trans. Image Processing, vol. 17, no. 10, pp. 1876-1890, Oct. 2008.

[7] R. A. Ulichney, Digital Halftoning. MIT Press, 1987.

[8] T. Mitsa and K. J. Parker, "Digital halftoning using a blue noise mask," in Proc. of IEEE ICASSP, vol. 4, Apr. 1991, pp. 2809 2812.

[9] A. Glassner, Principles of Digital Image Synthesis. Morgan Kaufmann Publishers, 1995.

[10] M. Dippé and E. Wold, "Antialiasing through stochastic sampling," in Proc. of SIGGRAPH, 1985, pp. 69-78.

[11] D. Mitchell, "Generating antialiased images at low sampling densities," in Proc. of SIGGRAPH, 1987, pp. 65-72.

[12] J. I. J. Yellot., "Spectral consequences of photoreceptor sampling in the rhesus retina," Science, pp. 382-385, 1983.

[13] Y. Hel-Or, "The canonical correlations of color images and their use for demosaicing," HP Laboratories Israel, Tech. Rep. HPL-2003-164R1, Feb. 2004.

[14] M. McCool and E. Fiume, "Hierarchical Poisson disk sampling distributions," in Proc. of Graphics Interface, 1992, pp. 94105.

[15] L. Condat, "A generic variational approach for demosaicking from an arbitrary color filter array," in Proc. of IEEE ICIP, Nov. 2009.

[16] — "A new color filter array with optimal sensing properties," in Proc. of IEEE ICIP, Nov. 2009.

[17] J. B. Rodríguez, G. R. Arce, and D. L. .Lau, "Blue-noise multitone dithering," IEEE Trans. Image Processing, vol. 17, no. 8, pp. 245-267, Aug. 2008.

[18] M. F. Cohen, J. Shade, S. Hiller, and O. Deussen, "Wang tiles for image and texture generation," in Proc. of SIGGRAPH, 2003, pp. 287-294.

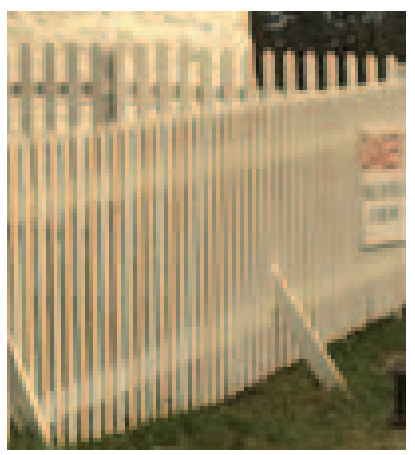

Reference image

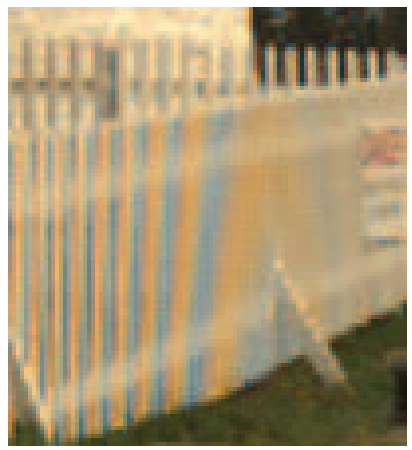

(a) Bayer

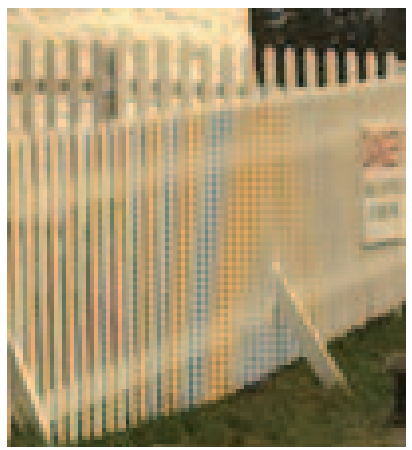

(c) Bayer

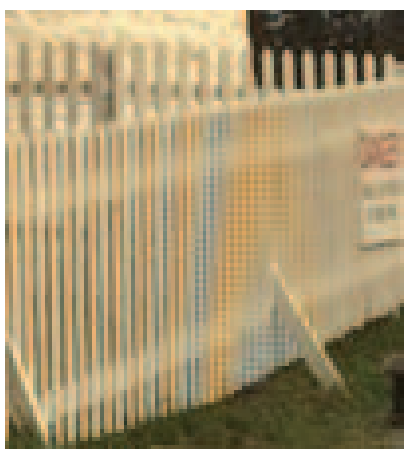

(e) Bayer

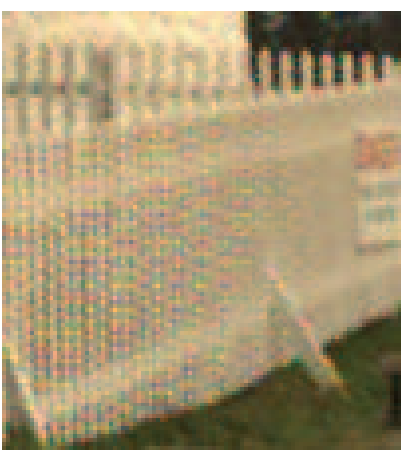

(b) Proposed

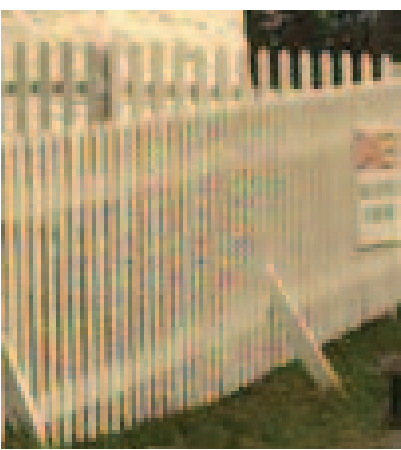

(d) Proposed

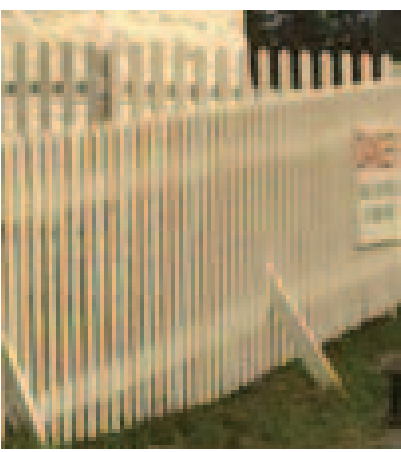

(f) Proposed
Fig. 2. Part of the demosaicked image lighthouse. The three rows correspond to the three demosaicking methods used: from top to bottom, bilinear, method of [3], method of [15]. 